\title{
Carnets
}

Revue électronique d'études françaises de l'APEF

Deuxième série - 6 | 2016

Exotopies de Barthes

\section{Du plaisir du texte à l'utilité de la littérature}

Dérive des Humanités aujourd'hui à partir de Barthes

\section{Isabel Fernandes}

\section{CpenEdition}

Journals

Édition électronique

URL : http://journals.openedition.org/carnets/693

DOI : $10.4000 /$ carnets.693

ISSN : 1646-7698

Éditeur

APEF

Référence électronique

Isabel Fernandes, « Du plaisir du texte à l'utilité de la littérature », Carnets [En ligne], Deuxième série - 6 |

2016, mis en ligne le 31 janvier 2016, consulté le 10 décembre 2020. URL : http://

journals.openedition.org/carnets/693; DOI : https://doi.org/10.4000/carnets.693

Ce document a été généré automatiquement le 10 décembre 2020.

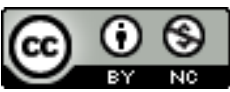

Carnets est mis à disposition selon les termes de la licence Creative Commons - Atribution - Pas

d'utilisation commerciale 4.0 International. 


\title{
Du plaisir du texte à l'utilité de la littérature
}

\author{
Dérive des Humanités aujourd'hui à partir de Barthes
}

\author{
Isabel Fernandes
}

«Le livre fait le sens, le sens fait la vie. » (Roland

Barthes, $1973: 51$ )

1 Mes propos d'aujourd'hui ne doivent pas être reçus comme ceux d'une spécialiste de l'œuvre de Barthes ; ils sont plutôt l'expression d'une admiration et d'appréciation que je voue à cet auteur et à l'héritage qu'il nous a légué.

2 Le Barthes structuraliste a accompagné mes années d'études : Le Degré zéro de l'écriture (1953), Mythologies (1957) et Critique et vérité (1966). Ce n'est qu'à la fin de mon cursus universitaire et quand j'ai moi-même commencé à enseigner que j'ai découvert le Barthes dit « poststructuraliste » et que, par le biais de cette découverte, je me suis graduellement aperçue d'un tournant épistémologique qui, peu de temps après, me révélerait les écrits d'un Jacques Derrida, d'un Michel Foucault ou d'un Jacques Lacan. C'est à cette époque que j'ai lu « La mort de l'auteur » (1968), Le Plaisir du texte (1973), et Fragments d'un discours amoureux (1977). Dans ces textes, se détachait clairement, au niveau du style, une fragmentation du discours révélatrice d'une écriture d'où se dégageait une respiration plus ample, une écriture plus liée au plaisir créatif de l'écrivain qu'à la performance docile de l'écrivant.

Dans ma thèse de doctorat, rédigée au cours des années 80 , je commence par faire une citation de Barthes, prise justement dans Le Plaisir du texte, un de mes textes préférés :

Texte veut dire Tissu; mais alors que jusqu'ici on a toujours pris ce tissu pour un produit, un voile tout fait, derrière lequel se tient, plus ou moins caché, le sens (la vérité), nous accentuons maintenant, dans le tissu, l'idée générative que le texte se fait, se travaille à travers un entrelacs perpétuel; perdu dans ce tissu - cette texture - le sujet s'y défait, telle une araignée qui se dissoudrait elle-même dans les sécrétions constructives de sa toile. (Barthes, 1973 : 100-101)

C'est par ce morceau que je voudrais commencer ses brèves remarques. Dans « La mort de l'auteur » on percevait déjà très clairement le début de ce que $\mathrm{M}$. $\mathrm{H}$. Abrams, remarquable 
intellectuel nord-américain récemment disparu, a appelé l'âge de la lecture (Age of Reading), une époque où l'attention était passée d'une approche du texte littéraire en tant que tel à l'importance accordée au rôle du lecteur (Abrams, 1989 : 365-66).

5 Auparavant, époque qui coïncidait grosso modo avec la première moitié du $\mathrm{xx}^{\mathrm{e}}$ siècle, Abrams a appelé l'âge de la critique (Âge of Criticism), l'époque comprenant tant le Formalisme Russe que le New Criticism anglophone et le Structuralisme de base française. La préoccupation avec les structures textuelles et les mécanismes qui régissent le fonctionnement interne du texte cède la place à la fuite vers l'extérieur à travers le lecteur et la lecture, selon un mouvement où l'on peut distinguer une volonté de se libérer d'une ambiance quelque peu claustrophobe. À mon avis, c'est dans Le Plaisir du texte bien plus clairement encore que dans «La mort de l'auteur » que devient visible le nouveau tournant: la disparition de la catégorie d'auteur en tant qu'institution et, surtout, la nouvelle centralité du lecteur et de la lecture, très visible dans l'extrait pris dans l'œuvre ultérieure, datée de 1973. Il reste très peu de l'« araignée-auteur » et c'est le lecteur, responsable souverain d'un "entrelacs perpétuel » à partir de la "toile-texte ", qui prend le devant. Sa tâche ne consiste plus à découvrir le sens ou la vérité cachés dans le texte/produit fini mais plutôt à révéler les potentialités d'un déploiement infini, autrement dit, sa productivité même, puisque le lecteur est perçu comme un rêveur qui, en dérive incessante, procède à l'« exploration du mot ", comme Barthes l'avait déjà reconnu quelques années avant, en Critique et vérité:

[U]ne œuvre est 'éternelle', non parce qu'elle impose un sens unique à des hommes différents, mais parce qu'elle suggère des sens différents à un homme unique, qui parle toujours la même langue symbolique à travers des temps multiples : l'œuvre propose, l'homme dispose.

Tout le lecteur sait cela, s'il veut bien ne pas se laisser intimider par les censures de la lettre: ne sent-il pas qu'il reprend contact avec un certain au-delà du texte, comme si le langage premier de l'œuvre développait en lui d'autres mots et lui apprenait à parler une seconde langue ? C'est ce qu'on appelle rêver. Mais le rêve a ses avenues, selon le mot de Bachelard, et ce sont ces avenues qui sont tracées devant le mot par la seconde langue de l'œuvre. La littérature est exploration du mot. (Barthes, 1966 : 51-52)

6 J'ai l'habitude d'utiliser cet extrait pour sensibiliser mes étudiants de première année à l'importance des mécanismes connotatifs au niveau de la lecture - ce que Barthes appelle, dans le fragment cité, la «seconde langue de l'œuvre »: comment les dérives de sens auxquelles consentent les mots du texte sont fondamentales et comment c'est par ces dérives que le texte appartient non plus à l'auteur, mais au lecteur. La même idée aura été menée par Jorge Luís Borges si l'on considère ce qu'il désigne par une "œuvre qui perdure » comme : « un miroir qui dévoile les traits du lecteur » (Borges, 1998: 772-774, ma traduction). Cette appropriation du texte par le lecteur nous intéresse non seulement parce qu'elle affranchit celui-ci de la critique établie mais surtout par l'envoûtement affectif et sensoriel qui naît entre lecteur et texte. Or c'est justement un rapport de ce genre que Barthes explore dans Le Plaisir du texte, quand il dit : « Le plaisir du texte, c'est ce moment où mon corps va suivre ses propres idées - car mon corps n'a pas les mêmes idées que moi » (Barthes, 1973: 30), déplaçant le plaisir au niveau de la physiologie, " d'une physiologie différente » (Barthes, 1973 : 49) et prenant le lecteur pour un « corpssujet » (Cf. Merleau-Ponty Merleau-Ponty, $1998: 114$ et suiv.).

7 Les allusions à caractère sensoriel/sensuel et affectif employées pour caractériser la relation de désir qui relie le lecteur et le texte vus comme deux corps qui se cherchent 
mutuellement, se succèdent le long de l'ouvrage. Si l'auteur est quelqu'un qui joue avec le corps de la mère, son objet de plaisir étant la langue maternelle, ce même texte, venu de l'auteur, tel un objet fétiche, "choisit » le lecteur qui, de son côté, cherche l'auteur. Paradoxal ? Pas du tout. - «Comme institution, l'auteur est mort», réaffirme Barthes (1973 : 45). Il est maintenant dépossédé du formidable pouvoir autrefois conféré par l'histoire littéraire mais il se trouve, tout de même, disséminé dans le texte ou " perdu dans ce tissu » et il laisse entrevoir sa présence par intermittence, devenant irrésistible au lecteur.

Barthes rattache la séduction du texte à un vertigineux plaisir verbal qui oblige le lecteur à tituber. Cela se produit par une sorte de " franciscanisme ", « tous les signifiants sont là et chacun fait mouche ; l'auteur (le lecteur) semble leur dire : je vous aime tous » (Barthes, 1973 : 17). Cette jubilation verbale suprême ne peut mener qu'à une évaluation solipsiste du texte qui empêche tout jugement critique - «pas de critique» (Barthes, 1973: 24). Barthes déclare : « le texte (il en est de même pour la voix qui chante) ne peut m'arracher que ce jugement, nullement adjectif : c'est ça! Et plus encore : c'est ça pour moi!» (ibid). L'allusion à "la voix qui chante", quoique parenthétique, n'est ni accidentelle ni négligeable, puisqu'elle se rapporte à l'importance des aspects sensoriels, du côté signifiant de la matière littéraire, dont on pourrait souligner la sonorité et son effet sur le lecteur. En perdant l'habitude de la lecture à haute-voix, on a perdu de vue une dimension cruciale du texte, plus précisément du poème, que le même Abrams désigne comme la quatrième dimension (Abrams, 2012). Selon lui, cette dimension se rapporte à la totalité des activités orales par lesquelles le poème se réalise quand il est récité, provoquant des sensations de mouvement, de forme, tactiles et autres encore ${ }^{1}-$ une dimension d'importance majeure puisque, d'après Abrams :

the oral actions that body forth the words of a poem, even when they remain below the level of awareness, may serve, in intricate and diverse ways, to interact with, confirm, and enhance the meanings and feelings that the words convey (Abrams, 2012: 3)

9 C'est ce que le critique nomme avec justesse : «[to] reembody [the poem] » (ibid). Quand il commente un poème de 1936 du poète britannique W. H. Auden, "On this Island», Abrams remarque, par exemple, que le lecteur est contraint de s'arrêter sur ce morceau :

- this island, now

The leaping light for your delight discovers.

Et, magistralement, il observe:

In representing the dazzle of the light (which seems to leap up from the moving waters that reflect it), Auden matches the delight he experiences in viewing the represented scene by the delight he evokes in our oral activities of verbalizing that representation - delight in the repeated utterance of the elastic l's, and in the evolution of the speech units, from 'leaping' to 'light' to 'delight' to 'discovers' ('the leaping light for your delight discovers'). (Abrams, $2012: 5$ )

11 Délectation (" delight ») est le mot-clé dans le poème et dans cet extrait, puisqu' il s'agit de l'irrépressible et inespéré plaisir qui se détache de la matérialité du texte sans que l'on ait toujours conscience qu'il vient de l'harmonie entre cette matière sensible et le sens qui la soutient et qui nous oblige à nous écrier : c'est ça! Ou, en une formulation analogue : impossible de le dire autrement! Chez Abrams, comme chez Barthes, c'est la matière physique du texte qui supporte le plaisir ${ }^{2}$, pouvant l'élever au seuil clandestin et imprévisible de la fruition, devenue réponse instable et indicible. 
12 Mais si au centre de l'œuvre de Barthes se trouvent les notions interdépendantes de plaisir et jouissance, on y trouve simultanément un élan de démocratisation de la lecture paisible :

Imaginer une esthétique (...) fondée jusqu'au bout (...) sur le plaisir du consommateur, quel qu'il soit, à quelque classe, à quelque groupe qu'il appartienne, sans acception de cultures et de langages: les conséquences seraient énormes, peut-être même déchirantes (...). (Barthes, 1973 : 94)

13 Je découvre dans une œuvre de 2008, Uses of Literature, de Rita Felski, une attitude également antisystème et anti-critique. Bien que sa pensée se trouve considérablement éloignée, du point de vue temporel et même théorique, de la position de Barthes (que, pourtant, elle évoque dans un des chapitres les plus intéressants de son travail, «Enchantement »), Felski prétend aussi libérer la littérature de la tutelle de la critique et de la théorie littéraires et la reconduire à une plus grande proximité avec le lectorat. S'inspirant surtout d'une attitude phénoménologique, comme celle adoptée par Paul Ricœur, l'auteur s'éloigne délibérément des théories de la lecture et autres modèles théorico-critiques proposés pendant les $\mathrm{xx}^{\mathrm{e}}$ et $\mathrm{xxI}^{\mathrm{e}}$ siècles et prône :

'Back to the things themselves' - was phenomenology's famous rallying cry: the insistence that we need to learn to see - to really see - what lies right under our nose. We are called on, in other words, to do justice to how readers respond to the words they encounter, rather than relying on textbook theories or wishful speculations about what reading is supposed to be. (Felski, 2008:17)

Ce que l'auteur propose est de prendre au sérieux ce qu'un lecteur quelconque (qui ne serait pas un expert : critique ou universitaire) cherche dans la littérature. Et elle conclut qu'il s'agit ici d'aspects que les théories littéraires récentes ont curieusement négligés.

Felski nomme les quatre chapitres fondamentaux de son œuvre d'après les motifs qui, selon elle, poussent et attirent le lecteur vers la littérature - ce sont: Reconnaissance, Connaissance, Enchantement et Choc. Commençons par deux d'entre eux: Reconnaissance et Connaissance. Ce que l'auteur veut nous transmettre, dans le premier cas, - Reconnaissance, c'est l'idée selon laquelle « les mérites potentiels de la littérature comme guide pour l'autoanalyse et la connaissance de soi » [« literature's potential merits as a guide to self-interpretation and self-understanding ( (Felski, 2008: 83)] sont en jeu. En d'autres termes, les textes littéraires que nous lisons nous amènent à un jugement intra-subjectif, déclenché par la complexité des formes de vie représentées dans l'œuvre. Par Connaissance, d'un autre côté, l'auteur se réfère à : « ce que la littérature révèle sur le monde au-delà de l'individu» ["what literature discloses about the world beyond the self» (ibid)], poussant le lecteur à un examen extra-subjectif. Mais si, par ces deux catégories, Felski s'éloigne des idées barthésiennes, elle se rapproche de lui, en revanche, à propos de Choc et Enchantement. Par le premier terme, l'auteur désigne une réaction à ce qui est surprenant, douloureux ou terrible, ce qui constitue un coup pour notre conscience et qui brise les cadres de référence qui nous sont familiers (Felski, $2008: 105$ ). En ce qui concerne le second, - Enchantement -, il concerne: « a sense of being so entirely caught up in an aesthetic object that nothing else seems to matter " (ibid.: 54). Il s'agirait d'un empoisonnement par lequel le lecteur cède au texte, en un abandon extatique, clairement marqué par l'érotisme (ibid. : 51).

Barthes, Abrams ou Felski nous font penser à ce que l'on pourrait sans doute appeler aussi le pouvoir de la littérature, de sa lecture et de son enseignement ${ }^{3}$, un pouvoir qui, comme nous l'avons vu, est complexe et va très au-delà de la surface de ce qui est représenté en 
elle, touchant de façon subtile et subliminaire l'homme qui lit comme un ensemble: intellect, corps, affects, émotions.

$\mathrm{Au}$ XXI ${ }^{\mathrm{e}}$ siècle, où l'on a tant parlé de crise des Humanités ${ }^{4}$, comment ne pas céder à la légitime tentation d'amplifier les formidables potentialités de la lecture des textes littéraires? Réagissant à l'incitation de Jean Starobinski dans son texte «Plaidoyer pour des 'Humanités médicales'", nous osons utiliser la littérature dans le cadre de notre projet « Récit \& Médecine », soit dans des cours de troisième cycle pour des étudiants en Médecine, soit en des groupes de lecture pour personnel médical et paramédical, étant donné que :

(...) [L]' approche 'humaniste' n'est ni la concurrente ni le substitut de la médecine scientifique et des moyens que celle-ci détient. Elle n'est surtout pas l'alliée de l'antiscience et de la superstition.

Les jeunes médecins ne gagneraient-ils pas quelques longueurs d'avance, si on leur mettait sous les yeux, au cours de leurs études, quelques pages de Balzac et Flaubert, de Manzoni et de Tolstoï, de Proust et de Virginia Woolf, ou encore de Tchékhov, Valéry, Kafka, Thomas Mann? (Starobinski, 2001 : 7-8)

Nous sommes convaincus que la sorte de détournement des textes littéraires que nous nous sommes permis d'organiser en adoptant des textes littéraires dans l'enseignement de notre cours interdisciplinaire de Médecine Narrative est en rapport avec ce type de propos. Nous parvenons, nous semble-t-il, à dynamiser des groupes de lecteurs en utilisant l'œuvre d'auteurs comme celui que nous célébrons aujourd'hui. Cette dérive contribue, selon nous, à l'enrichissement personnel et humain de nos étudiants et des professionnels de santé avec qui nous travaillons, les aidant à affiner leur sensibilité, à partager la parole et la perspective d'autrui et, grâce à ceci, à humaniser leur rapport avec les patients.

\section{BIBLIOGRAPHIE}

ABRAMS, M. H. (1989). Doing Things with texts. NY, London: W. W. Norton.

ABRAMS, M. H. (2012). The Fourth Dimension of a poem. NY, London: W. W. Norton.

BARTHES, Roland (1966). Critique et vérité. Paris : Seuil.

BARTHES, Roland (1973). Le Plaisir du texte. Paris : Seuil.

BORGES, Jorge Luís ([1952] 1998). « O Primeiro Wells », in Jorge Luís Borges. Obras completas

1952-1972. Trad. José Colaço Barreiros. S. 1. : Círculo de Leitores, vol. II, pp. 772-74.

FELSKI, Rita (2008). Uses of literature. Malden and Oxford: Blackwell Publishing.

FERNANDES, Isabel (2013). « Os Estudos Literários no séc. XXI : o passado próximo, a crise e o próximo futuro », in Júlio Carlos Viana (et al). A Scholar for all seasons : volume de homenagem a João de Almeida Flor. Lisboa : Centro de Estudos Anglísticos da Universidade de Lisboa/ Departamento de Estudos Anglísticos da Faculdade de Letras da Universidade de Lisboa, pp. 349-366. 
HARPHAM, Geoffrey Galt (2005). «Politics, professionalism, and the pleasures of reading ", Daedalus, 134, 3, pp. 68-75 [on-line]. [disponible le 15-08-06]

http://www.mitpressjournals.org/doi/abs/10.1162/0011526054622088\#.VyKAS9QrLMx

HUGHES, Ted ([1967] 1969). Poetry in the Making: an anthology of poems and programmes from « listening and writing ". Londres, Boston : Faber \& Faber.

MERLEAU-PONTY, Maurice (1998). Phénoménologie da la perception. Paris : Gallimard.

STAROBINSKI, Jean (2001). « Plaidoyer pour des 'Humanités médicales' in Gérard Danou (éd.).

Littérature et médecine, ou les pouvoirs du récit. Paris : Bibliothèque publique d'information.

\section{NOTES}

1. À ce propos voir Ted Hughes (1969: 17-18).

2. Dans un autre passage Barthes évoque ce qu'il désigne comme "grain de la voix » et qui est en rapport avec ce qu'Abrams défend ci-dessus.

3. Geoffrey Galt Harpham affirme: «More so than any other subject, literature lends itself to powerful teaching " (2005: 68-75).

4. À propos de cette crise, voir Isabel Fernandes (2013).

\section{RÉSUMÉS}

Dans mon article je propose de revenir à Barthes et de reprendre en particulier Le Plaisir du Texte (1973), qui marque un tournant épistémologique dans l'œuvre vers le poststructuralisme. Barthes y souligne l'importance de la matière signifiante et la dérive qu'elle permet ; il insiste aussi sur la position antisystème envers l'académie et la critique institutionnelle. Je rapproche les analyses barthésiennes de certaines prises de positions récentes dans le monde anglophone, notamment celles de M. H. Abrams (2012) et de Rita Felski (2008). Ces auteurs attirent à leur façon l'attention sur le(s) pouvoir(s) du texte littéraire et sa lecture. Je termine par quelques mots sur le projet en Médecine Narrative, que je placerai également sous l'égide barthésienne.

In my article I propose to go back to Barthes and in particular to Le Plaisir du Texte (1973), which signals an epistemological turn towards post-structuralism in his work. Barthes emphasizes the impact of the text's formal/aural features and the digressions (dérives) these features enable; he insists also on his anti-system standing against academy and institutionalised criticism. I associate his approaches to literature with recent positions in the English-speaking world, namely by M. H. Abrams (2012) and Rita Felski (2008). In different ways both authors call attention to the power(s) of the literary text and its reading. I conclude with a few words on our interdisciplinary project in Narrative Medicine, which will be placed under the aegis of Roland Barthes. 
INDEX

Mots-clés : plaisir, lecture, médecine narrative, Abrams (M. H.), Felski (Rita)

Keywords : delight, reading, narrative medicine, Abrams (M. H.), Felski (Rita)

\section{AUTEUR}

ISABEL FERNANDES

ULICES - University of Lisbon Centre for English Studies Universidade de Lisboa isacrfernandes@sapo.pt 